\title{
A new measurement of the leading hadronic corrections to the muon $\mathbf{g - 2}{ }^{\star}$
}

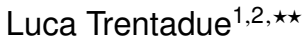 \\ ${ }^{1}$ Dipartimento di Fisica e Scienze della Terra "Macedonio Melloni" \\ Universitá di Parma, Parma \\ 2 INFN Sezione di Milano Bicocca, Milano, Italy
}

\begin{abstract}
A novel approach to determine the leading hadronic corrections to the muon $g$-2 is proposed. It consists in a measurement of the effective electromagnetic coupling in the space-like region. This method may become feasible at flavor factories resulting in a determination potentially competitive with the dispersive approach via time-like data.
\end{abstract}

\section{Introduction}

The motivation of this work [1] is due to a long-standing discrepancy between experiment and the Standard Model (SM) prediction for $a_{\mu}$, the muon anomalous magnetic moment. For this reason the hadronic corrections have been kept under close scrutiny [2-5]. The hadronic contribution represents the largest uncertainty of the SM value and is comparable with the experimental one. When the new results from the $g$-2 experiments at Fermilab and J-PARC will reach the unprecedented precision of 0.14 parts per million (or better) [6-8], the uncertainty of the hadronic corrections will become the main limitation of this formidable test of the SM.

Vacuum Polarization makes $\alpha_{e m}$ running assuming a well defined effective value at any scale. Vacuum polarization and the effective charge are defined by:

$$
e^{2} \rightarrow e^{2}\left(q^{2}\right)=\frac{e^{2}}{1+\left(\Pi\left(q^{2}\right)-\Pi(0)\right)}
$$

and

$$
\alpha\left(q^{2}\right)=\frac{\alpha(0)}{1-\Delta \alpha} \Delta \alpha=-\operatorname{Re}\left(\Pi\left(q^{2}\right)-\Pi(0)\right) .
$$

$\Delta \alpha$ takes contributions from leptonic and hadronic elementary states among these the non-perturbative $\Delta \alpha_{\text {had }}$

$$
\begin{gathered}
\Delta \alpha=\Delta \alpha_{l e p t}+\Delta \alpha_{\text {had }}+\Delta \alpha_{\text {top }} \\
\Delta \alpha_{\text {had }}^{(5)}\left(M_{z}^{2}\right)=-\frac{\alpha M_{z}^{2}}{3 \pi} \operatorname{Re} \int_{4 m_{\pi}^{2}}^{\infty} d s \frac{R(s)}{s\left(s-M_{z}^{2}-i \epsilon\right)}
\end{gathered}
$$

${ }^{\star}$ This talk is based on a work done in collaboration with C. Carloni Calame, M. Passera, and G. Venanzoni.

$\star \star$ e-mail: luca.trentadue@unipr.it 
A direct measurement of the running of $\alpha_{e m}(s / t)$ in space/time-like regions can show the running of $\alpha_{e m}$. It can provide a test of duality far away form resonances and ita has been done in the past by few experiments at $e^{+} e^{-}$colliders by comparing well known QED processes with some reference (either data or MonteCarlo one) normalization:

$$
\left(\frac{\alpha\left(q^{2}\right)}{\alpha\left(q_{0}^{2}\right)}\right)^{2} \simeq \frac{N_{\text {signal }}\left(q^{2}\right)}{N_{\text {norm }}\left(q_{0}^{2}\right)} .
$$

$N_{\text {signal }}$ can be any QED process as muon pairs production or others similar ones and $N_{\text {norm }}$ can be the Bhabha process, a pure QED channel as $\gamma \gamma$ pair production as well as theory, or any other reference process. $q_{0}$ represents a scale chosen as starting point for the running.

\section{$2 a_{m u}^{H L O}$ calculation}

The leading-order hadronic contribution to the muon $g$ - 2 is given by the well-known formula $[5,12]$

$$
a_{\mu}^{\mathrm{HLO}}=\frac{\alpha}{\pi^{2}} \int_{0}^{\infty} \frac{d s}{s} K(s) \operatorname{Im} \Pi_{\mathrm{had}}(s+i \epsilon)
$$

where $\Pi_{\text {had }}(s)$ is the hadronic part of the photon vacuum polarization, $\epsilon>0$,

$$
K(s)=\int_{0}^{1} d x \frac{x^{2}(1-x)}{x^{2}+(1-x)\left(s / m_{\mu}^{2}\right)}
$$

is a positive kernel function, and $m_{\mu}$ is the muon mass. As the total cross section for hadron production in low-energy $e^{+} e^{-}$annihilations is related to the imaginary part of $\Pi_{\text {had }}(s)$ via the optical theorem, the dispersion integral in eq. (6) is computed integrating experimental time-like $(s>0)$ data up to a certain value of $s[3,16,17]$. The high-energy tail of the integral is calculated using perturbative QCD [18].

Alternatively, if we exchange the $x$ and $s$ integrations in eq. (6) we obtain [19]

$$
a_{\mu}^{\mathrm{HLO}}=\frac{\alpha}{\pi} \int_{0}^{1} d x(x-1) \bar{\Pi}_{\mathrm{had}}[t(x)],
$$

where $\bar{\Pi}_{\text {had }}(t)=\Pi_{\text {had }}(t)-\Pi_{\text {had }}(0)$ and

$$
t(x)=\frac{x^{2} m_{\mu}^{2}}{x-1}<0
$$

is a space-like squared four-momentum. If we invert eq. (9), we get $x=(1-\beta)\left(t / 2 m_{\mu}^{2}\right)$, with $\beta=$ $\left(1-4 m_{\mu}^{2} / t\right)^{1 / 2}$, and from eq. (8) we obtain

$$
a_{\mu}^{\mathrm{HLO}}=\frac{\alpha}{\pi} \int_{-\infty}^{0} \bar{\Pi}_{\mathrm{had}}(t)\left(\frac{\beta-1}{\beta+1}\right)^{2} \frac{d t}{t \beta} .
$$

Equation (10) has been used for lattice QCD calculations of $a_{\mu}^{\mathrm{HLO}}$ [20]; while the results are not yet competitive with those obtained with the dispersive approach via time-like data, their errors are expected to decrease significantly in the next few years [21]. 
The effective fine-structure constant at squared momentum transfer $q^{2}$ is defined above and is $\Delta \alpha\left(q^{2}\right)=-\operatorname{Re} \bar{\Pi}\left(q^{2}\right)$. The purely leptonic part, $\Delta \alpha_{\text {lep }}\left(q^{2}\right)$, can be calculated order-by-order in perturbation theory - it is known up to three loops in QED [22] (and up to four loops in specific $q^{2}$ limits [23]). As $\operatorname{Im} \bar{\Pi}\left(q^{2}\right)=0$ for negative $q^{2}$, eq. (8) can be rewritten in the form [24]

$$
a_{\mu}^{\mathrm{HLO}}=\frac{\alpha}{\pi} \int_{0}^{1} d x(1-x) \Delta \alpha_{\mathrm{had}}[t(x)] .
$$

Equation (11), involving the hadronic contribution to the running of the effective fine-structure constant at space-like momenta, can be further formulated in terms of the Adler function [25], defined as the logarithmic derivative of the vacuum polarization, which, in turn, can be calculated via a dispersion relation with time-like hadroproduction data and perturbative QCD [24, 26]. We will proceed differently, proposing to calculate eq. (11) by measurements of the effective electromagnetic coupling in the space-like region. The hadronic contribution to the running of $\alpha$ in the space-like region, $\Delta \alpha_{\text {had }}(t)$ (see eq. (3)), can be extracted comparing Bhabha scattering data to Monte Carlo (MC) predictions. The LO Bhabha cross section receives contributions from $t$ - and $s$-channel photon exchange amplitudes. At NLO in QED, it is customary to distinguish corrections with an additional virtual photon or the emission of a real photon (photonic NLO) from those originated by the insertion of the vacuum polarization corrections into the LO photon propagator (VP). let us consider a few simple points. In fig. 1 (left) we plot the integrand $(1-x) \Delta \alpha_{\text {had }}[t(x)]$ of eq. (11) using the output of the routine hadr5n12 [29] (which uses time-like hadroproduction data and perturbative QCD). The range $x \in(0,1)$ corresponds to $t \in(-\infty, 0)$, with $x=0$ for $t=0$. The peak of the integrand occurs at $x_{\text {peak }} \simeq 0.914$ where $t_{\text {peak }} \simeq-0.108 \mathrm{GeV}^{2}$ and $\Delta \alpha_{\text {had }}\left(t_{\text {peak }}\right) \simeq 7.86 \times 10^{-4}$ (see fig. 1 (right)). Such relatively low $t$ values
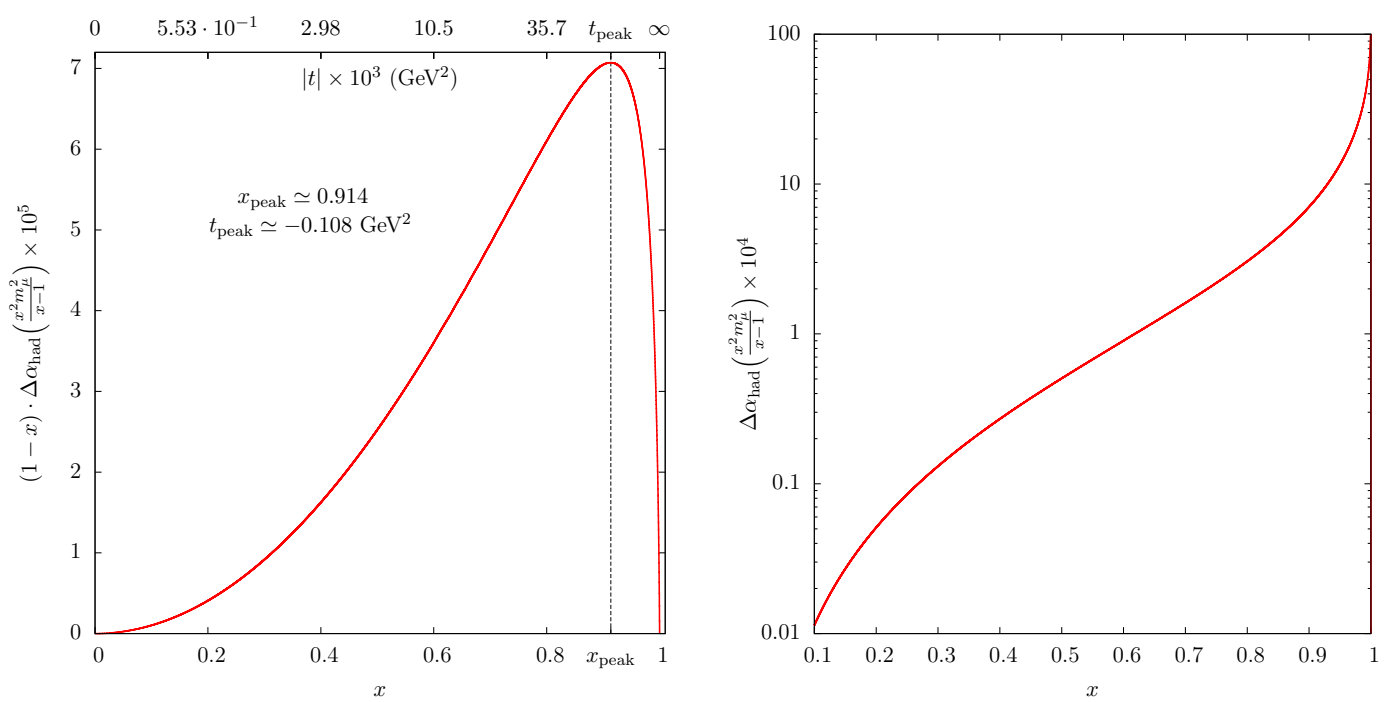

Figure 1. Left: The integrand $(1-x) \Delta \alpha_{\text {had }}[t(x)] \times 10^{5}$ as a function of $x$ and $t$. Right: $\Delta \alpha_{\text {had }}[t(x)] \times 10^{4}$.

can be explored at $e^{+} e^{-}$colliders with center-of-mass energy $\sqrt{s}$ around or below $10 \mathrm{GeV}$ where

$$
t=-\frac{s}{2}(1-\cos \theta)\left(1-\frac{4 m_{e}^{2}}{s}\right)
$$


$\theta$ is the electron scattering angle and $m_{e}$ is the electron mass. Depending on $s$ and $\theta$, the integrand of eq. (11) can be measured in the range $x \in\left[x_{\min }, x_{\max }\right]$, as shown in fig. 2 (left). Note that to span low $x$ intervals, larger $\theta$ ranges are needed as the collider energy decreases. In this respect, $\sqrt{s} \sim 3 \mathrm{GeV}$ appears to be very convenient, as an $x$ interval $[0.30,0.98]$ can be measured varying $\theta$ between $\sim 2^{\circ}$ and $28^{\circ}$. It is also worth remarking that data collected at flavor factories, such as DAФNE (Frascati), VEPP-2000 (Novosibirsk), BEPC-II (Beijing), PEP-II (SLAC) and SuperKEKB (Tsukuba), and possibly at a future high-energy $e^{+} e^{-}$collider, like FCC-ee (TLEP) [30] or ILC [31], can help to cover different and complementary $x$ regions.

Furthermore, given the smoothness of the integrand, values outside the measured $x$ interval may be interpolated with some theoretical input. In particular, the region below $x_{\min }$ will provide a relatively small contribution to $a_{\mu}^{\text {HLO }}$, while the region above $x_{\max }$ may be obtained by extrapolating the curve from $x_{\max }$ to $x=1$, where the integrand is null, or using perturbative QCD.
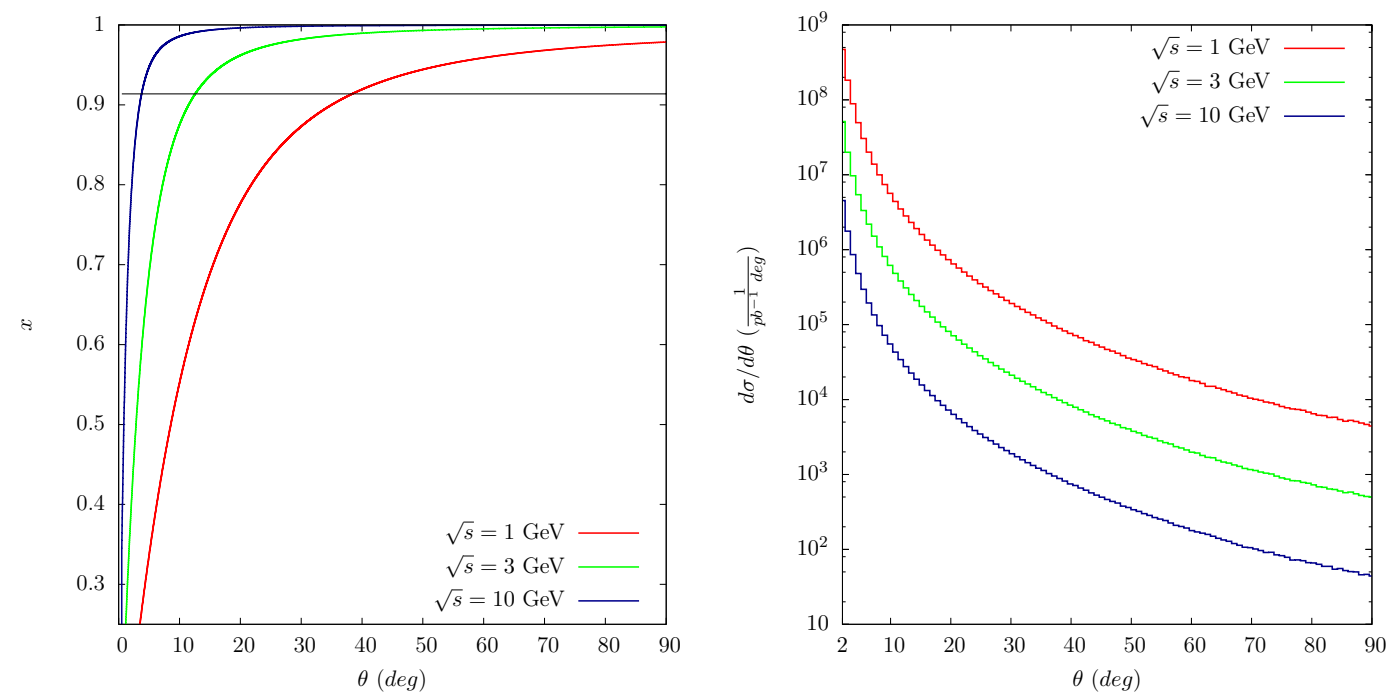

Figure 2. Left: Ranges of $x$ values as a function of the electron scattering angle $\theta$ for three different center-ofmass energies. The horizontal line corresponds to $x=x_{\text {peak }} \simeq 0.914$. Right: Bhabha differential cross section obtained with BabaYaga [27] as a function of $\theta$ for the same three values of $\sqrt{s}$ in the angular range $2^{\circ}<\theta<90^{\circ}$.

The analytic dependence of the MC Bhabha predictions on $\alpha(t)$ (and, in turn, on $\Delta \alpha_{\text {had }}(t)$ ) is not trivial, and a numerical procedure has to be devised to extract it from the data.This was not the case for example in [13,14]: there $\alpha(t)$ was extracted from Bhabha data in the very forward region at LEP, where the $t$ channel diagrams are by far dominant and $\alpha(t)$ factorizes (see for example [15]). In formulae, we have to find a function $\alpha(t)$ such that

$$
\left.\frac{d \sigma}{d t}\right|_{\mathrm{data}}=\left.\frac{d \sigma}{d t}(\alpha(t), \alpha(s))\right|_{\mathrm{MC}},
$$

where we explicitly kept apart the dependence on the time-like $\operatorname{VP} \alpha(s)$ because we are only interested in $\alpha(t)$. We emphasise that, in our analysis, $\alpha(s)$ is an input parameter. Being the Bhabha cross section in the forward region dominated by the $t$-channel exchange diagrams, we checked that the present $\alpha(s)$ uncertainty induces in this region a relative error on the $\theta$ distribution of less than $\sim 10^{-4}$ (which is part of the systematic error). 
We propose to perform the numerical extraction of $\Delta \alpha_{\text {had }}(t)$ from the Bhabha distribution of the $t$ Mandelstam variable. The idea is to let $\alpha(t)$ vary in the MC sample around a reference value and choose, bin by bin in the $t$ distribution, the value that minimizes the difference with data. The procedure can be sketched as follows:

1. choose a reference function returning the value of $\Delta \alpha_{\text {had }}(t)$ (and hence $\alpha(t)$ ) to be used in the MC sample, we call it $\bar{\alpha}(t)$;

2. for each generated event, calculate $N$ MC weights by rescaling $\bar{\alpha}(t) \rightarrow \bar{\alpha}(t)+\frac{i}{N} \delta(t)$, where $i \in[-N, N]$ and $\delta(t)$ is for example the error induced on $\bar{\alpha}(t)$ by the error on $\Delta \alpha_{\text {had }}(t)$. Being done on an event by event basis, the full dependence on $\alpha(t)$ of the MC differential cross section can be kept;

3. for each bin $j$ of the $t$ distribution, compare the experimental differential cross section with the MC predictions and choose the $i_{j}$ which minimizes the difference;

4. $\bar{\alpha}\left(t_{j}\right)+\frac{i_{j}}{N} \delta\left(t_{j}\right)$ will be the extracted value of $\alpha\left(t_{j}\right)$ from data in the $j^{t h}$ bin. $\Delta \alpha_{\text {had }}\left(t_{j}\right)$ can then be obtained through the relation between $\alpha(t)$ and $\Delta \alpha_{\text {had }}(t)$.

We finally find, for each bin $j$ of the $t$ distribution,

$$
\left.\frac{d \sigma}{d t}\right|_{j, \text { data }}=\left.\frac{d \sigma}{d t}\left(\bar{\alpha}(t)+\frac{i_{j}}{N} \delta(t), \alpha(s)\right)\right|_{j, \mathrm{MC}} \cdot
$$

We remark that the algorithm does not assume any simple dependence of the cross section on $\alpha(t)$, which can in fact be general, mixing $s, t$ channels and higher order radiative corrections, relevant (or not) in different $t$ domains.

In order to test our procedure, we perform a pseudo-experiment: we generate pseudo-data using the parameterization $\Delta \alpha_{\text {had }}^{I}(t)$ of refs. [17, 32] and check if we can recover it by inserting in the MC the (independent) parameterization $\Delta \alpha_{\text {had }}^{I I}(t)$ (corresponding to $\bar{\alpha}(t)$ of eq. 14) of ref. [29] by means of the method described above. For this exercise, we use the generator BabaYaga in its most complete setup, generating events at $\sqrt{s}=1.02 \mathrm{GeV}$, requiring $10^{\circ}<\theta_{ \pm}<170^{\circ}, E_{ \pm}>0.4 \mathrm{GeV}$ and an acollinearity cut of $15^{\circ}$. We choose $\delta(t)$ to be the error induced on $\alpha(t)$ by the $1-\sigma$ error on $\Delta \alpha_{\text {had }}(t)$, which is returned by the routine of ref. [29], we set $N=150$, and we produce distributions with 200 bins. We note that in the present exercise $\alpha(s)$ and all the radiative corrections both in the pseudo-data and in the MC samples are exactly the same, because we are interested in testing the algorithm rather than assessing the achievable accuracy, at least at this stage.

In fig. 3, $\Delta \alpha_{\text {had }}^{\text {extr }}$ is the result extracted with our algorithm, corresponding to the minimizing set of $i_{j}$ : the figure shows that our method is capable of recovering the underlying function $\Delta \alpha_{\text {had }}(t)$ inserted into the "data". As the difference between $\Delta \alpha_{\text {had }}^{I}$ and $\Delta \alpha_{\text {had }}^{\text {extr }}$ is hardly visible on an absolute scale, in fig. 3 all the functions have been divided by $\Delta \alpha_{\text {had }}^{I I}$ to display better the comparison between $\Delta \alpha_{\text {had }}^{I}$ and $\Delta \alpha_{\text {had }}^{\text {extr }}$.

In order to assess the achievable accuracy on $\Delta \alpha_{\text {had }}(t)$ with the proposed method, we remark that the $\mathrm{LO}$ contribution to the cross section is quadratic in $\alpha(t)$, thus we have

$$
\frac{1}{2} \frac{\delta \sigma}{\sigma} \simeq \frac{\delta \alpha}{\alpha} \simeq \delta \Delta \alpha_{\mathrm{had}}
$$

Equation (15) relates the absolute error on $\Delta \alpha_{\text {had }}$ with the relative error on the Bhabha cross section. Let us stress here that: 


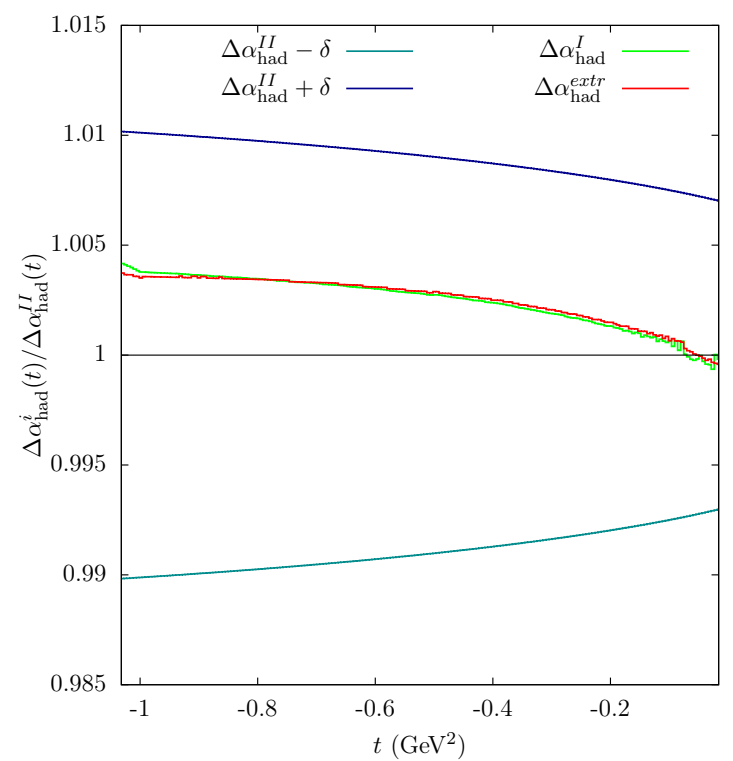

Figure 3. The extracted function $\Delta \alpha_{\text {had }}^{\text {extr }}(t)$ compared to the function $\Delta \alpha_{\text {had }}^{I}(t)$ used in the pseudo-data (see text). The functions $\Delta \alpha_{\text {had }}^{I I}(t) \pm \delta(t)$ are shown to display the range spanned by the MC samples. All functions have been divided by $\Delta \alpha_{\text {had }}^{I I}(t)$. The tiny difference between $\Delta \alpha_{\text {had }}^{I}$ and $\Delta \alpha_{\text {had }}^{\text {extr }}$ is due to the binning discretization.

1. From the theoretical point of view, the present accuracy of the MC predictions [28] is at the level of about $0.05 \%$, which implies that the precision that our method can, at best, set on $\Delta \alpha_{\text {had }}(t)$ is $\delta \Delta \alpha_{\text {had }}(t) \simeq 2 \cdot 10^{-4}$. Any further improvement requires the inclusion of the NNLO QED corrections into the MC codes (see Ref. [28]).

2. Experimentally a measurement of $a_{\mu}^{\mathrm{HLO}}$ from space-like data competitive with the current time-like evaluations would require an $O(1 \%)$ accuracy. Statistical considerations show that a $3 \%$ fractional accuracy on the $a_{\mu}^{\text {HLO }}$ integral can be obtained by sampling the integrand $(1-x) \Delta \alpha_{\text {had }}[t(x)]$ in $\sim 10$ points around the $x$ peak with a fractional accuracy of $10 \%$. Given the value of $O\left(10^{-3}\right)$ for $\Delta \alpha_{\text {had }}$ at $x=x_{\text {peak }}$, this implies that the cross section must be known with relative accuracy of $\sim 2 \times 10^{-4}$. Such a statistical accuracy, although challenging, can be obtained at flavor factories, as shown in fig. 2 (right). With an integrated luminosity of $O(1)$, $O(10), O(100) \mathrm{fb}^{-1}$ at $\sqrt{s}=1,3$ and $10 \mathrm{GeV}$, respectively, the angular region of interest can be covered with a $0.01 \%$ accuracy per degree. The experimental systematic error must match the same level of accuracy.

A source of experimental systematic errors comes from the machine luminosity, which can be normalized by calculating a theoretical cross section in principle not depending on $\Delta \alpha_{\text {had }}$. We devise two possible options for the normalization process:

1. To use the $e^{+} e^{-} \rightarrow \gamma \gamma$ process, which has no dependence on $\Delta \alpha_{\text {had }}$, at least up to NNLO order;

2. To use the Bhabha process at $t \sim 10^{-3} \mathrm{GeV}^{2}(x \sim 0.3)$, where the dependence on $\Delta \alpha_{\text {had }}$ is of $O\left(10^{-5}\right)$ and can be safely neglected. 
It is worth quoting that a detailed analysis of the systematic errors involved in the measurement of the luminosity has been carried out at LEP by the OPAL collaboration reaching the final accuracy of $O\left(10^{-4}\right)[14,33]$.

\section{Conclusions}

We presented a novel approach to determine the leading hadronic correction to the muon $g$-2 based on measurements of the running of $\alpha(t)$ in the space-like region.

This approach, even if challenging, may become feasible by using data collected at present flavor factories as well as those at future high-energy $e^{+} e^{-}$colliders ${ }^{1}$.

The proposed determination can become competitive with the accuracy of the present results obtained with the dispersive approach via time-like data.

An alternative formula for $a_{\mu}^{H L O}$ in the space-like region has been studied in detail. It emphasizes low values of $t \leq 1 G e V^{2}$ which can be explored at low energies machines.

We have also argued that this requires a measurement of the Bhabha cross section, at relatively small angles, with an accuracy of the order $10^{-4}$ or better.

Reaching such an accuracy demands a dedicated experimental and theoretical work for the next few years.

\section{Acknowledgements}

I wish to thank Fulvia de Fazio and Pietro Colangelo for inviting me to this lively Workshop.

\section{References}

[1] C. Carloni Calame, M. Passera, G. Venanzoni, L. Trentadue, Phys. Lett. B 746 (2015 ) 325-329.

[2] G.W. Bennett et al. [Muon g-2 Collaboration], Phys. Rev. D 73 (2006) 072003.

[3] F. Jegerlehner, A. Nyffeler, Phys. Rept. 477 (2009) 1.

[4] T. Blum et al., arXiv:1311.2198 [hep-ph]; K. Melnikov, A. Vainshtein, Springer Tracts Mod. Phys. 216 (2006) 1; M. Davier, W.J. Marciano, Ann. Rev. Nucl. Part. Sci. 54 (2004) 115; M. Passera, J. Phys. G 31 (2005) R75; M. Knecht, Lect. Notes Phys. 629 (2004) 37.

[5] F. Jegerlehner, "The anomalous magnetic moment of the muon," Springer Tracts Mod. Phys. 226, 2008.

[6] J. Grange et al. [Muon g-2 Collaboration], arXiv:1501.06858 [physics.ins-det].

[7] G. Venanzoni [Muon g-2 Collaboration], arXiv:1411.2555 [physics.ins-det].

[8] N. Saito [J-PARC g-2/EDM Collaboration], AIP Conf. Proc. 1467 (2012) 45.

[9] G. Venanzoni, Nuovo Cim. C 037 (2014) 02, 165; G. Venanzoni, Frascati Phys. Ser. 54 (2012) 52.

[10] G.V. Fedotovich [CMD-2 Collaboration], Nucl. Phys. Proc. Suppl. 181-182 (2008) 146.

\footnotetext{
${ }^{1}$ The possibility of using a fixed target $\mu e$ scattering process is also under investigation [34].
} 
[11] G. Colangelo, M. Hoferichter, A. Nyffeler, M. Passera, P. Stoffer, Phys. Lett. B 735 (2014) 90.

[12] C. Bouchiat, L. Michel, J. Phys. Radium 22 (1961) 121; L. Durand, Phys. Rev. 128 (1962) 441 [Erratum-ibid. 129 (1963) 2835]; M. Gourdin, E. De Rafael, Nucl. Phys. B 10 (1969) 667.

[13] A.B. Arbuzov, D. Haidt, C. Matteuzzi, M. Paganoni and L. Trentadue, Eur. Phys. J. C 34 (2004) 267.

[14] G. Abbiendi et al. [OPAL Collaboration], Eur. Phys. J. C 45 (2006) 1.

[15] A.B. Arbuzov, V.S. Fadin, E.A. Kuraev, L.N. Lipatov, N.P. Merenkov, L. Trentadue, Nucl. Phys. B485 (1997) 457-502.

[16] M. Davier, A. Hoecker, B. Malaescu, Z. Zhang, Eur. Phys. J. C 71 (2011) 1515 [Erratum-ibid. C 72 (2012) 1874].

[17] K. Hagiwara, R. Liao, A.D. Martin, D. Nomura, T. Teubner, J. Phys. G 38 (2011) 085003.

[18] R.V. Harlander, M. Steinhauser, Comput. Phys. Commun. 153 (2003) 244.

[19] B.E. Lautrup, A. Peterman, E. de Rafael, Phys. Rept. 3 (1972) 193.

[20] C. Aubin, T. Blum, Phys. Rev. D 75 (2007) 114502; P. Boyle, L. Del Debbio, E. Kerrane, J. Zanotti, Phys. Rev. D 85 (2012) 074504; X. Feng, K. Jansen, M. Petschlies, D.B. Renner, Phys. Rev. Lett. 107 (2011) 081802; M. Della Morte, B. Jager, A. Juttner, H. Wittig, JHEP 1203 (2012) 055.

[21] T. Blum, M. Hayakawa, T. Izubuchi, PoS LATTICE 2012 (2012) 022.

[22] M. Steinhauser, Phys. Lett. B 429 (1998) 158.

[23] P.A. Baikov, K.G. Chetyrkin, J.H. Kuhn and C. Sturm, Nucl. Phys. B 867 (2013) 182; C. Sturm, Nucl. Phys. B 874 (2013) 698; P.A. Baikov, A. Maier, P. Marquard, Nucl. Phys. B 877 (2013) 647.

[24] F. Jegerlehner, in Proceedings of "Fifty years of electroweak physics: a symposium in honour of Professor Alberto Sirlin's 70th birthday", New York University, 27-28 October 2000, J. Phys. G 29 (2003) 101.

[25] S.L. Adler, Phys. Rev. D 10 (1974) 3714.

[26] S. Eidelman, F. Jegerlehner, A. L. Kataev, O. Veretin, Phys. Lett. B 454 (1999) 369.

[27] G. Balossini, C.M. Carloni Calame, G. Montagna, O. Nicrosini, F. Piccinini, Nucl. Phys. B 758 (2006) 227.

[28] S. Actis et al., Eur. Phys. J. C 66 (2010) 585.

[29] S. Eidelman, F. Jegerlehner, Z. Phys. C 67 (1995) 585; F. Jegerlehner, Nucl. Phys. Proc. Suppl. 181-182 (2008) 135.

[30] M. Bicer et al. [TLEP Design Study Working Group Collaboration], JHEP 1401 (2014) 164 [arXiv:1308.6176 [hep-ex]].

[31] G. Aarons et al. [ILC Collaboration], Physics at the ILC, arXiv:0709.1893 [hep-ph].

[32] K. Hagiwara, A.D. Martin, D. Nomura, T. Teubner, Phys. Lett. B 649 (2007) 173; Phys. Rev. D 69 (2004) 093003.

[33] G. Abbiendi et al. [OPAL collaboration], Eur. Phys. J. C14 (2000) 373-425.

[34] G. Abbiendi, C. M. Carloni Calame, U. Marconi, C. Matteuzzi, G. Montagna, O. Nicrosini, M. Passera, F. Piccinini, R. Tenchini, L. Trentadue, G. Venanzoni et al., hep-ex, arXiv:1609.08987. 